\title{
Aktuelles aus der italienischsprachigen Schweiz
}

\author{
Nicola Gianinazzi
}

Anlässlich der Jubiläumsveranstaltung 40 Jahre ASP veröffentlichen wir diesen kurzen Beitrag über unsere Geschichte.

In den letzten zehn Jahren hat die ASP auch die Entstehung ihrer Sektion der italienischen Schweiz erlebt: Sie wurde auf Anregung ihres damaligen Direktors Emil Stutz und meiner Person mit dem Ziel geschaffen, die Assoziation auf ihrer italienischsprachigen Seite $\mathrm{zu}$ revitalisieren. Bereits die erste "Gründungsversammlung» im Jahr 2010 verzeichnete die starke Beteiligung von rund 20 Mitgliedern (fast alle italienischsprachigen Mitglieder). Das gemütliche Abendessen im Anschluss an das Treffen bestätigte den offiziellen Beginn dieser Reise endgültig.

Die Sektion brachte der ASP mehrere neue Mitglieder, darunter zahlreiche - aber nicht ausschliesslich - Jugendliche, ein Kollektivmitglied und erneut einen Delegierten in der kantonalen Beratungskommission für PsychologInnen und PsychotherapeutInnen.

Seit über einem Jahr werden ihre Beratungs-, Netzwerk-, Ausbildungs- und Kulturvermittlungstätigkeiten in der gestrafften Form einer Delegation für die italienischsprachigen Schweiz durchgeführt.

\section{Aus der italienischen Schweiz}

Wir beraten regelmässig PsychologInnen und PsychotherapeutInnen sowie gelegentlich inund ausländische Bildungseinrichtungen und andere private und öffentliche Institutionen.

Was die innerassoziative Arbeit betrifft, so besteht sie hauptsächlich aus der Vermittlung zwischen regionalen und nationalen Wirklichkeiten, der Beratung sowie der Überprüfung und Übersetzung von Informationen oder vorwiegend technischen Texten.

Bei der Organisation der Weiterbildung arbeiten wir weiterhin erfolgreich mit dem «Istituto Ricerche di Gruppo» (Institut für Gruppenforschung) zusammen, so dass wir jederzeit ein breites Spektrum an qualitativ hochstehenden Kursen garantieren können. Die Kurse können somit gemäss dem ASP-Integral-Konzept im Rahmen des Nachdiplomstudiums ausgewählt werden.

Zudem bin ich seit 2018 Mitglied des Redaktionsteams Psychotherapie-Wissenschaft. Die italienische Schweiz und das italienische Panorama werden in unserem thematischen Magazin sichtbarer und stärker präsent sein.
Die Zusammenarbeit mit HochschullehrerInnen verschiedener Universitäten und Fakultäten im Tessin geht weiter. Diese interessante Öffentlichkeitsarbeit ist nützlich und notwendig, um sowohl die Ausbildungsvorschläge als auch die Beiträge unserer Zeitschriften zu bereichern. Im letzteren Bereich wird auch die redaktionelle Zusammenarbeit mit der Zeitschrift Psicoterapia e scienze uma$n e$ (Psychotherapie und Sozialwissenschaften) vertieft, als deren Co-Direktor Paolo Migone amtet.

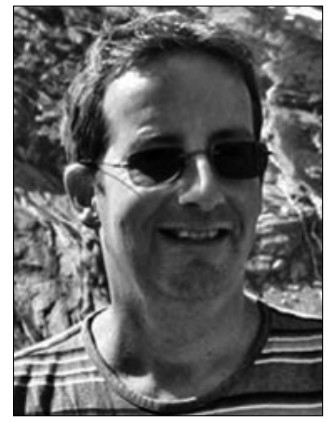

\section{Das psychotherapeutische Umfeld im Tes- sin und jenseits der Grenze}

Im Tessin wurde - im Rahmen einer internationalen Zusammenarbeit mit mehreren ASP-Mitgliedern, akademischen ForscherInnen und sonstigen PartnerInnen - eine Website für die Forschung in den Bereichen Gesundheitsmedizin, Psychotherapie, Psychologie und Humanwissenschaft im Allgemeinen eingerichtet. Ihre Aufgabe ist es, Forschungsprojekte zu erkennen, zu planen und durchzuführen sowie psychologische und psychosoziale Fragen zu untersuchen, die für die im Kanton Tessin lebenden BürgerInnen von Bedeutung sind.

Unsere Projekte haben keinen ausschliesslich kognitiven Zweck, auch wenn Wissen für uns als ForscherInnen einen fundamentalen Wert hat. Ziel ist es, psychologische und psychosoziale Fragen und Phänomene zu untersuchen, die für die im Kanton Tessin lebenden Menschen von besonderer Relevanz sind. Bei der Entwicklung unserer Projekte schenken wir den spezifischen Besonderheiten, aber vor allem den Bedürfnissen unseres Territoriums besondere Beachtung. Die Aufgabe von «Psychology Research Ticino» besteht nicht nur im Beobachten und Katalogisieren von psychologischen und sozialen Phänomenen, die sich im Tessin entwickeln. Es geht uns vielmehr darum, das aus unseren Studien gewonnene Wissen zu nutzen, um Bildungs-, Förder- und Unterstützungsprogramme für die Gesundheit vorzuschlagen und aufzubauen, die in der Region tatsächlich umsetzbar sind. Sie sollen dazu beitragen, die Lebensqualität der in unserem Kanton lebenden BürgerInnen zu verbessern. Der Auftrag von «Psychology Research Ticino» lautet zusammengefasst:

- Förderung der wissenschaftlichen Forschung im psychologischen und psychosozialen Umfeld des Tessins 
- Aktive Beteiligung der TessinerInnen an den Forschungsprojekten der «Psychology Research Ticino» durch Förderung eines Klimas der Zusammenarbeit, der aktiven Beteiligung und der Eigenverantwortung in Gesundheitsfragen

- Vermittlung der Ergebnisse der von der «Psychology Research Ticino» geförderten Forschungsprojekte mit dem Ziel, die BürgerInnen über Gesundheits- und Wellnessthemen zu informieren, die für die Lebensqualität des Einzelnen wichtig sind

- Verbreitung der wichtigsten Neuigkeiten in Bezug auf Psychologie und Psychotherapie gegenüber Laien im Gesundheitswesen - in verständlicher Sprache, aber strenger wissenschaftlicher Form

- Schulung des im Tessin tätigen medizinischen Fachpersonals hinsichtlich der Aspekte, die mit unserer Forschungstätigkeit verbunden sind
Die wichtigsten Anwendungsbereiche für «Psychology Research Ticino» lauten:

- Beurteilung und Förderung der psychischen Gesundheit und des psychologischen Wohlbefindens der im Kanton Tessin lebenden Bevölkerung

- Untersuchung und Prävention von Risikoverhalten bei den im Kanton lebenden Jugendlichen und jungen Erwachsenen

- Beurteilung der Probleme und Anpassungsschwierigkeiten der im Kanton Tessin lebenden Jugendlichen und jungen Erwachsenen

- Beurteilung der Probleme und Anpassungsschwierigkeiten der im Kanton Tessin lebenden Familien

Link zur Website von «Psychology Research Ticino»: https://www.psychologyresearchticino.ch

Nicola Gianinazzi ist Vorstandsmitglied und Delegierter für die italienische Schweiz.

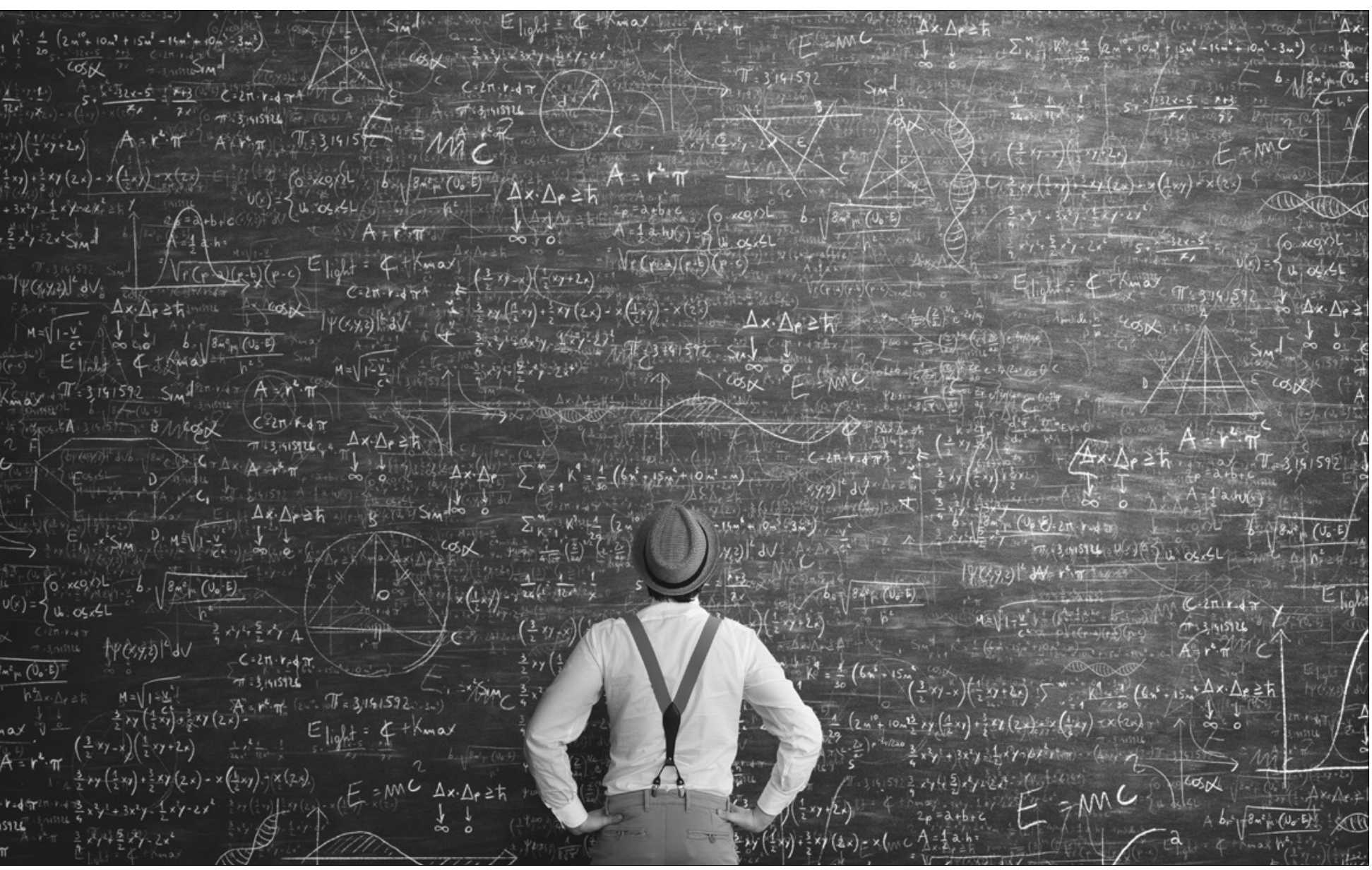

